\section{Tendência da mortalidade por diabetes melito no Brasil: 1950 a 2000}

\author{
Mortality trends by the diabetes mellitus in Brazil: 1950 to 2000
}

Eduarda Ângela Pessoa Cesse', Eduardo Freese de Carvalho', Wayner Vieira de Souza' ${ }^{1}$ Carlos Feitosa Luna'

\section{RESUMO}

Objetivo: Analisar a tendência da mortalidade por diabetes melito em capitais brasileiras. Métodos: Foi analisada a tendência temporal da mortalidade por diabetes melito em capitais brasileiras, a partir das Razões de Mortalidade Padronizadas construídas com dados de óbitos

'Departamento de Saúde Coletiva (NESC), Centro de Pesquisas Aggeu Magalhães (CPqAM), Fundação Oswaldo Cruz (Fiocruz), Recife, PE, Brasil

Correspondência para: Eduarda Ângela Pessoa Cesse NESC, CPqAM, Fiocruz Campus da Universidade Federal de Pernambuco

Av. Moraes Rego, s/n-Cidade Universitária

50670-420 - Recife, PE, Brasil educesse@cpqam.fiocruz.br

Recebido em 6/Set/2008 Aceito em 15/Jun/2009

dos anuários estatísticos do IBGE e do Sistema de Informação de Mortalidade do Ministério da Saúde, no período de 1950 a 2000. Resultados: Foi observado crescimento da mortalidade proporcional e da Razão de Mortalidade Padronizada por diabetes na maioria das capitais. São Paulo (SP) se destacou por apresentar mortalidades proporcionais expressivas e constantes ao longo da série, particularmente a partir de 1960. Quanto às Razões de Mortalidade Padronizadas nessa capital, bem como em Belo Horizonte (MG), onde foi observada a oscilação desses indicadores tendendo a diminuição nos últimos anos, não foram verificadas tendências significantes. Conclusões: A tendência da mortalidade por diabetes é crescente na maioria das capitais com exceção de São Paulo e Belo Horizonte, que apresentaram padrões diferentes das demais capitais. Ara Bras Endocrinol Metab. 2009;53(6):760-6

Descritores

Diabetes melito; mortalidade; tendências

\begin{abstract}
Objective: To analyze mortality trend by diabetes mellitus in Brazilian capitals. Methods: It was analyzed mortality temporal trend by diabetes mellitus in Brazilian capitals, from the death data of the statistic directory of IBGE and the System of Mortality Information of the Ministry of Health, in the period from 1950 to 2000. Results: It was observed the growth of proportional mortality and the Standard Mortality Ratio by diabetes in most of the capitals. São Paulo (SP) outstood for presenting expressive and constant proportional mortalities along the series, particularly from 1960. As to the Standard Mortality Ratio in this capital, as well as in Belo Horizonte (MG), where it was noticed the oscillation of these indicators tending to decrease in the last years, it was not observed significant trends. Conclusions: Mortality trend by diabetes is increasing in most of the capitals, with the exception of São Paulo and Belo Horizonte, which have shown different patterns from the other capitals. Arq Bras Endocrinol Metab. 2009;53(6):760-6

Keywords

Diabetes mellitus; mortality; trends
\end{abstract}

\section{INTRODUÇÃO}

o longo dos séculos, o Brasil tem experimentado Aacentuadas transformações, reflexos da história social, econômica e cultural de constituição das suas diversas regiões, Estados e municípios, num cenário de desigualdades que persiste na atualidade. Nesse contexto, o século 20 assume particular importância. Por influência do crescimento do comércio internacional, instalam-se formas capitalistas de produção, trabalho e consumo, e é com base nessa dinâmica que o processo de industrialização e de urbanização se desenvolve de maneira mais acelerada (1).

Dessa forma, e sob influência das mudanças nos modos de vida, fruto dos intensos processos de industrialização e urbanização, verifica-se o aumento da obesidade e do sedentarismo, que constituem importantes fatores de risco para inúmeras doenças. Vale salientar que, do 
conjunto de fatores demográficos, econômicos, sociais e de mudanças nutricionais que interferem no padrão de morbimortalidade no país, o aumento da longevidade populacional chama particular atenção, por sua ocorrência de modo acelerado, aumentando a probabilidade de expressão das enfermidades crônicas, tais como o diabetes melito tipo 2 (DMT2), que geralmente se manifestam em idades mais avançadas (2).

A ocorrência do diabetes melito é um fenômeno universal e afeta populações de países em todos os estágios de desenvolvimento. Dessa forma, observa-se o aumento da frequência da figuração da doença nas estatísticas de mortalidade, tanto como causa básica ou contributória, especialmente associada a doenças renais, cardiovasculares e cerebrovasculares (3).

São essas associações que carregam o essencial da gravidade da doença e representam uma enorme sobrecarga para a Saúde Pública $(4,5)$. Previsões indicam que 250 milhões de pessoas deverão apresentar a doença até 2020 (6).

Dos casos de diabetes diagnosticados, $90 \%$ são de "diabetes tipo 2" e dizem respeito a uma doença pouco sintomática ou assintomática, que ocorre principalmente em pessoas acima dos 50 anos, com obesidade ou sobrepeso e com histórico familiar da doença. Essas características a diferenciam do "diabetes tipo l", que apresenta sintomas mais definidos e acomete mais crianças ou jovens, além de requerer a insulina como tratamento permanente ao longo da vida (7).

No Brasil, o estudo mais abrangente sobre a prevalência do diabetes foi realizado em nove capitais em 1988. A prevalência estimada, ajustada por idade, variou de $5,2 \%$ a $9,7 \%$ nos diferentes centros urbanos avaliados. As capitais das regiões Sul e Sudeste apresentaram as maiores taxas (8).

Dados mais recentes de uma campanha nacional para detecção de casos suspeitos de diabetes realizada entre os meses de março e abril de 2001, em todas as regiões do país, identificaram 2,9 milhões de suspeitos $(14,66 \%)$, sendo, aproximadamente, 1 milhão, entre eles, de hipertensos e portadores de diabetes (9).

Quanto à mortalidade, Franco (3) relata que, de um modo geral, os dados obtidos mediante atestados de óbito subestimam a importância do diabetes como causa básica e, frequentemente, essa enfermidade não aparece no atestado de óbito de diabéticos, principalmente nos idosos, nos quais estão presentes várias doenças crônicas.

Diante desse contexto, este artigo teve por objetivo analisar a tendência da mortalidade por diabetes melito em capitais brasileiras, considerando o período de 1950 a 2000, como forma de observar as mudanças na tendência da mortalidade por esse agravo.

\section{MÉTODOS}

Foi realizado um estudo de série temporal. A casuística do estudo compreendeu o universo conhecido dos óbitos por diabetes melito, como causa básica, em capitais brasileiras, nos anos censitários de 1950 a 2000 e no ano de 1996, quando ocorreu a recontagem da população.

Foram utilizados dados oficiais de óbitos publicados nos anuários estatísticos do Brasil e divulgados pelo Instituto Brasileiro de Geografia e Estatística (IBGE) (10), bem como dados do Sistema de Informação de Mortalidade (SIM) do Departamento de Informática do Sistema Único de Saúde (Datasus)/Ministério da Saúde. Para tanto, considerou-se a Classificação Internacional de Doenças (CID) que, atualmente, encontrase em sua décima revisão e as revisões dessa codificação realizadas no período considerado no estudo. Os dados populacionais censitários tiveram como fonte primária o IBGE.

Foi calculada a mortalidade proporcional (MP) por diabetes melito nas capitais brasileiras, nos anos censitários de 1950 a 2000 e do ano de 1996 em relação ao total de óbitos ocorridos nas referidas capitais no período considerado no estudo. Foi calculada a variação proporcional por diabetes melito nas capitais brasileiras, a partir da MP no último ano de análise (2000) e da MP no primeiro ano de análise (1950), considerando a equação: variação proporcional $=($ MP 2000/MP 1950 $)-1$.

Em seguida, foi calculada a taxa bruta de mortalidade (TBM), por 100 mil habitantes, no mesmo período. Para controlar o efeito das diferentes estruturas etárias das capitais brasileiras ao longo do período de análise, foi realizada a padronização das TBM por diabetes melito, por 100 mil habitantes. Foi empregada a técnica de padronização indireta, uma vez que até 1970 era desconhecida a distribuição dos óbitos por diabetes por faixas etárias. Tal técnica foi utilizada para verificar se o número de óbitos esperados (OEs) era diferente (maior ou menor) do que o observado sem a eliminação do efeito "idade" sobre as populações das capitais do estudo. Foram utilizadas como padrões a estrutura etária do Brasil no ano de 2000 e a distribuição de suas taxas brutas específicas para o diabetes melito. Em seguida, foi calculada a Razão de Mortalidade Padronizada (RMP), 
a partir da razão entre os óbitos observados $(\mathrm{OOb})$ e os OEs nas capitais do país.

Para verificar a tendência temporal da mortalidade por diabetes melito nas capitais brasileiras, foi feita a análise de tendência linear das RMP.

Foram estimados modelos de regressão linear simples, definidos como: $\mathrm{Y}=\alpha+\beta$ ANO, sendo $\alpha$ o coeficiente médio no período analisado e $\beta$ o incremento (acréscimo ou decréscimo) médio no período.

Utilizaram-se na análise o coeficiente angular, que se refere ao incremento de óbitos (acréscimo ou decréscimo), e o coeficiente de determinação, que indica a capacidade de explicação do modelo. Todas as decisões foram tomadas considerando o nível de significância estatística de $5 \%$.

Para elaboração e análise do banco de dados, foram utilizados os softwares TabWin 32, Statistical Package for the Social Sciences (SPSS) 8.0 e Excel 2000.

O estudo não apresentou implicações ético-morais por utilizar dados secundários e agrupados, nos quais não constaram informações que pudessem identificar os indivíduos. No entanto, o projeto original do qual resultou este artigo foi submetido e obteve a aprovação do Comitê de Ética em Pesquisa (CEP) do Centro de Pesquisas Aggeu Magalhães (CPqAM) da Fundação Oswaldo Cruz (Fiocruz), em Recife (PE).

\section{RESULTADOS}

A Tabela 1 mostra a MP e a variação proporcional da mortalidade por diabetes melito, em capitais brasileiras, no período de 1950 a 2000. As MP se apresentaram crescente ao longo do período de análise em praticamente todas as capitais analisadas. Destacam-se as MP verificadas em Natal (RN), Salvador (BA) e Belo Horizonte (MG), que apresentaram um declínio entre os anos censitários de 1991 e 2000. Por outro lado, as MP por diabetes verificadas em São Paulo (SP) foram as que se apresentaram mais expressivas e mais constantes ao longo da série histórica analisada, particularmente a partir de 1960. As maiores variações proporcionais da mortalidade no período foram verificadas em Teresina (PI), Recife (PE) e Natal $(55,1,27$ e 21,7\%, respectivamente).

A Tabela 2 traz os resultados referentes ao comportamento das RMP por diabetes melito e à análise de tendência temporal da mortalidade em capitais brasileiras no período de 1950 a 2000.

Observam-se RMP crescentes na maioria das capitais, ao longo do período de análise, principalmente a partir da década de 1970, a exemplo do verificado para Manaus (AM) e Belém (PA) na região Norte. Na região Nordeste, destacam-se as capitais São Luís (MA), Teresina, Fortaleza (CE) e Aracaju (SE), por apresentarem RMP crescentes

\begin{tabular}{|c|c|c|c|c|c|c|c|c|c|}
\hline Região & Capital & 1950 & 1960 & 1970 & 1980 & 1991 & 1996 & 2000 & Variação proporcional* \\
\hline \multirow{2}{*}{ Norte } & Manaus & 0,14 & 0,22 & 0,27 & 0,43 & 1,91 & 2,52 & 3,12 & 20,8 \\
\hline & Belém & 0,29 & 0,38 & 0,61 & 0,95 & 2,74 & 3,04 & 3,74 & 11,7 \\
\hline \multirow{9}{*}{ Nordeste } & São Luís & 0,59 & 1,07 & 0,29 & 1,60 & 3,45 & 3,68 & 4,56 & 6,7 \\
\hline & Teresina & 0,10 & 0,50 & 0,50 & 1,00 & 2,38 & 3,57 & 5,55 & 55,1 \\
\hline & Fortaleza & 0,21 & 0,12 & 0,63 & 1,01 & 2,17 & 2,89 & 3,67 & 16,6 \\
\hline & Natal & 0,23 & 0,36 & 0,81 & 1,24 & 5,35 & 5,50 & 5,32 & 21,7 \\
\hline & João Pessoa & 0,53 & 0,79 & 0,97 & 1,49 & 3,49 & 3,79 & 5,47 & 9,3 \\
\hline & Recife & 0,16 & 0,65 & 1,59 & 2,29 & 3,26 & 3,83 & 4,53 & 27,0 \\
\hline & Maceió & 0,45 & 0,74 & 1,37 & 2,17 & 3,68 & 4,33 & 4,95 & 10,0 \\
\hline & Aracaju & 0,44 & 0,67 & 1,35 & 2,50 & 3,90 & 3,83 & 7,01 & 15,0 \\
\hline & Salvador & 0,37 & 0,46 & 1,31 & 1,48 & 3,84 & 4,95 & 4,62 & 11,6 \\
\hline \multirow{4}{*}{ Sudeste } & Belo Horizonte & 0,49 & 0,48 & 0,54 & 1,84 & 3,70 & 2,62 & 2,82 & 4,8 \\
\hline & Vitória & 0,55 & 0,39 & 0,52 & 1,00 & 2,38 & 2,42 & 4,43 & 7,1 \\
\hline & Rio de Janeiro & 0,53 & 0,72 & 1,50 & 3,65 & 3,72 & 4,34 & 4,77 & 8,1 \\
\hline & São Paulo & 0,79 & 2,13 & 2,22 & 2,49 & 2,86 & 2,87 & 3,65 & 3,6 \\
\hline \multirow{3}{*}{ Sul } & Curitiba & 0,60 & 0,55 & 1,08 & 1,37 & 2,49 & 2,83 & 3,82 & 5,4 \\
\hline & Florianópolis & 0,46 & 0,38 & 0,27 & 1,54 & 1,77 & 3,79 & 4,49 & 8,7 \\
\hline & Porto Alegre & 0,61 & 0,97 & 1,91 & 1,32 & 3,01 & 3,36 & 3,76 & 5,2 \\
\hline
\end{tabular}

Fonte: Elaboração própria a partir de dados do IBGE e do Datasus/Ministério da Saúde.

* Variação proporcional = (MP 2000/MP 1950) -1. 


\begin{tabular}{|c|c|c|c|c|c|c|c|c|c|c|c|}
\hline Região & Capital & 1950 & 1960 & 1970 & 1980 & 1991 & 1996 & 2000 & $\beta$ & valor de $p$ & R2 (\%) \\
\hline \multirow{2}{*}{ Norte } & Manaus & 0,21 & 0,28 & 0,23 & 0,35 & 1,02 & 1,23 & 1,26 & 0,023 & 0,005 & 81,8 \\
\hline & Belém & 0,35 & 0,35 & 0,38 & 0,60 & 1,03 & 1,03 & 1,18 & 0,018 & 0,001 & 88,8 \\
\hline \multirow{9}{*}{ Nordeste } & São Luís & 0,50 & 0,94 & 0,20 & 0,84 & 1,48 & 1,42 & 1,44 & 0,021 & 0,038 & 61,2 \\
\hline & Teresina & 0,07 & 0,36 & 0,30 & 0,57 & 0,58 & 0,88 & 1,70 & 0,023 & 0,020 & 69,2 \\
\hline & Fortaleza & 0,45 & 0,18 & 0,63 & 0,59 & 0,68 & 1,00 & 0,97 & 0,013 & 0,014 & 73,4 \\
\hline & Natal & 0,41 & 0,41 & 0,67 & 0,56 & 1,55 & 1,72 & 1,32 & 0,026 & 0,010 & 76,8 \\
\hline & João Pessoa & 0,49 & 0,51 & 0,72 & 0,84 & 1,21 & 1,36 & 1,50 & 0,021 & $<0,001$ & 94,5 \\
\hline & Recife & 0,21 & 0,58 & 1,04 & 1,22 & 1,28 & 1,36 & 1,43 & 0,023 & 0,001 & 91,2 \\
\hline & Maceió & 0,49 & 0,69 & 1,21 & 1,53 & 1,72 & 1,66 & 1,76 & 0,026 & $<0,001$ & 93,6 \\
\hline & Aracaju & 0,47 & 0,58 & 0,52 & 1,79 & 1,41 & 1,52 & 2,29 & 0,033 & 0,011 & 75,9 \\
\hline & Salvador & 0,41 & 0,42 & 0,83 & 0,96 & 1,47 & 1,86 & 1,39 & 0,027 & 0,002 & 87,4 \\
\hline \multirow{4}{*}{ Sudeste } & Belo Horizonte & 0,43 & 0,32 & 0,32 & 1,02 & 1,33 & 0,84 & 0,71 & 0,013 & 0,102 & 44,4 \\
\hline & Vitória & 0,47 & 0,23 & 0,40 & 0,44 & 0,98 & 0,82 & 1,22 & 0,016 & 0,020 & 69,3 \\
\hline & Rio de Janeiro & 0,40 & 0,41 & 0,67 & 1,43 & 1,25 & 1,48 & 1,31 & 0,023 & 0,005 & 82,3 \\
\hline & São Paulo & 0,54 & 1,12 & 1,01 & 1,07 & 0,96 & 1,01 & 1,05 & 0,005 & 0,228 & 27,4 \\
\hline \multirow{3}{*}{ Sul } & Curitiba & 0,41 & 0,34 & 0,62 & 0,66 & 0,87 & 0,92 & 1,05 & 0,013 & $<0,001$ & 92,9 \\
\hline & Florianópolis & 0,26 & 0,17 & 0,10 & 0,60 & 0,44 & 1,15 & 1,02 & 0,018 & 0,025 & 66,8 \\
\hline & Porto Alegre & 0,42 & 0,50 & 0,63 & 0,48 & 0,90 & 1,01 & 0,96 & 0,012 & 0,006 & 80,2 \\
\hline
\end{tabular}

Fonte: elaboração própria a partir de dados do IBGE e do Datasus/Ministério da Saúde.

e oscilantes no período. Em João Pessoa (PB), Recife e Maceió (AL), as RMP apresentaram crescimento mais contínuo ao longo do período de análise. Já em Natal e Salvador, apesar das RMP se apresentarem crescentes, observou-se o declínio destas no final do período analisado.

$\mathrm{Na}$ região Sudeste, destacam-se Belo Horizonte e São Paulo. A primeira por apresentar RMP crescentes, porém em queda acentuada a partir de 1991. A segunda por apresentar RMP elevadas e estáveis a partir da década de 1960 até o final do período analisado.

$\mathrm{Na}$ região Sul, as RMP oscilaram em Porto Alegre (RS) e Florianópolis (SC) e foram mais constantes em Curitiba (PR).

Quanto à análise de tendência das séries históricas de mortalidade por diabetes melito nas capitais brasileiras, verifica-se que, com exceção de Belo Horizonte e São Paulo, que não mostraram tendências significativas, as demais capitais apresentaram tendências crescentes da mortalidade $(\mathrm{p}<0,05)$. Nessas capitais, o coeficiente de determinação $\left(R^{2}\right)$ foi de $60 \%$ e $90 \%$, respectivamente, demonstrando uma boa explicação do modelo de tendência da série de mortalidade por diabetes melito.

\section{DISCUSSÃO}

Conhecer o padrão de adoecimento e morte de uma população é útil para a proposição de políticas públicas e para a avaliação, gestão e planejamento de ações de promoção e prevenção realizadas pelos Serviços de Saúde. Para tanto, é necessário o desenvolvimento de estudos que enfoquem a tendência histórica de morbimortalidade.

Um dos principais desafios para a realização desses estudos no Brasil é a insuficiência de dados contínuos e sistematizados ao longo de décadas, principalmente no que se refere à morbidade por doenças crônicas não transmissíveis (DCNT), tais como o diabetes melito, na medida em que inexiste um monitoramento sistemático para esses agravos no plano nacional.

Destaca-se que o sistema de vigilância e monitoramento das DCNT ainda não está devidamente desenvolvido e implantado na maioria dos Estados brasileiros. Quando seu funcionamento for efetivo, este deverá ser operado a partir de dados de mortalidade, morbidade e de inquéritos de fatores de risco. Para tanto, deverá ser desencadeado a partir de sistemas já em funcionamento, como o SIM, o Sistema de Informação Hospitalar (SIH) e o Sistema de Informação sobre Hipertensão e Diabetes (SISHIPERDIA) (11). Se devidamente implantado, o sistema de vigilância e monitoramento das DCNT poderá fornecer informações importantes, que trarão subsídios para a tomada de decisão no âmbito da prevenção e controle dessas enfermidades.

Vale salientar que a informação gerada no âmbito da atenção básica sobre a morbidade por hipertensão 
e diabetes pelo SISHIPERDIA (9) apresenta limitações quanto à abrangência e cobertura, uma vez que se encontra desigualmente implantado nos Programas de Saúde da Família dos municípios. Consequentemente, os dados gerados não são uniformes, dificultando ou até impossibilitando comparações.

Dessa forma, a opção pela análise de tendência histórica de mortalidade por diabetes melito foi motivada pela ausência de dados para a construção de uma série histórica de morbidade por esse agravo nas capitais brasileiras. No entanto, torna-se oportuno referir o desconhecimento da estrutura de mortalidade por faixa etária nas capitais brasileiras, no período de 1950 a 1970, bem como as intercorrências nos censos realizados no período da análise (1950 a 2000), as quais comprometeram a qualidade dos dados populacionais.

Torna-se oportuno, ainda, registrar que a opção de trabalhar pela reconstrução de séries de mortalidade nas capitais procurou minimizar as limitações relacionadas à insuficiência de dados ao longo de décadas, tendo em vista o fato de que, nessas localidades, há a possibilidade concreta de uma informação de melhor qualidade. No entanto, é preciso considerar que, nas capitais, existe a possibilidade real de invasão de óbitos, motivada pela concentração de recursos assistenciais quando comparadas às grandes dificuldades enfrentadas pelos demais municípios brasileiros nesse aspecto.

Como consequência das limitações apontadas, verifica-se a existência de poucos e localizados estudos de tendência histórica de mortalidade por diabetes melito, com resgate de dados para além da série já disponibilizada pelo Ministério da Saúde, a partir 1980, sendo praticamente inexistentes aqueles que mostram o seu padrão de ocorrência, considerando o conjunto das capitais brasileiras.

Vale salientar que, durante a construção das séries históricas de mortalidade por diabetes melito nas capitais brasileiras, observou-se uma grande oscilação dos dados de óbito. No entanto, apesar dos problemas apontados, Laurenti e cols. (12) enfatizaram que são as estatísticas de mortalidade as que mais têm sido utilizadas para a análise de tendência no Brasil e, do ponto de vista qualitativo, têm exatidão e fidedignidade semelhantes as de qualquer outro país de longa tradição na elaboração dessas estatísticas.

A partir da reconstituição de séries históricas de mortalidade por diabetes melito, enquanto causa básica de óbito, num período de 50 anos, foi possível observar que a MP, por essa causa, apresenta um importante crescimento ao longo da segunda metade do século 20 em praticamente todas as capitais analisadas, em todas as regiões brasileiras (Tabela 1 ).

Apesar da importância desses achados, comparações entre maior ou menor magnitude da MP entre as capitais brasileiras ou regiões geográficas não fornecem informações sobre o comportamento da mortalidade na população pela causa específica. Também uma tendência, seja ela crescente ou decrescente e mesmo observada proporcionalmente, não é acompanhada de uma tendência similar das taxas de mortalidade pela causa medidas na população.

A análise da tendência da mortalidade por diabetes a partir das RMP também se revelou crescente na quase totalidade das capitais (Tabela 2). Mais uma vez, a oscilação dos dados sugere influência da qualidade duvidosa dos dados de óbito e de população.

Esses achados corroboram a análise que autores vêm realizando acerca do comportamento do diabetes melito, levando-os a referir a existência de uma epidemia desse agravo em diferentes regiões do planeta (13), sob influência da ampliação da longevidade populacional e do crescimento das taxas de obesidade (14). No Brasil, o diabetes melito representa a terceira causa isolada de mortalidade entre todas na atualidade (15).

Assumindo padrão diferente, destacam-se Belo Horizonte e São Paulo, que não apresentaram tendências definidas. Belo Horizonte apresentou RMP crescentes até 1991, seguido de importante declínio a partir desse ano, e São Paulo apresentou RMP altas e constantes, desde 1960. O padrão observado em São Paulo sugere que, possivelmente, nessa capital, tenha surgido um conjunto de características associadas aos processos de urbanização e industrialização de forma mais expressiva ainda na primeira metade do século 20 , as quais influenciaram o surgimento do diabetes melito, também, de forma mais precoce em relação às capitais de outras regiões do país.

As capitais das regiões Nordeste, Sudeste e Sul apresentaram tendências mais completas e definidas quando comparadas às capitais das regiões Norte e CentroOeste - esta última, inclusive, não apresentou nenhuma capital com série completa de dados no período do estudo, fato que pode estar relacionado às condições de constituição das capitais e à qualidade das informações.

O padrão verificado por meio de séries históricas nas capitais permite identificar que o nível de saúde dessas localidades pode ser explicado a partir de características específicas de determinação. Tais características se cons- 
tituem em fatos que espelham um padrão de mortalidade regional historicamente configurado. Esse padrão é fruto das transições epidemiológica e demográfica verificadas no país, entendidas como a expressão dinâmica das relações políticas, econômicas e sociais que ocorreram histórica e permanentemente no interior da sociedade (16).

Na presente situação brasileira, na qual têm sido observadas melhorias em indicadores de morbidade e mortalidade ao longo das últimas décadas, a inexistência de políticas que interfiram na intensidade dessas mudanças, particularmente para regiões e grupos sociais com os piores níveis socioeconômicos, significa a manutenção e mesmo a ampliação das desigualdades existentes.

Os resultados do estudo realizado por Laurenti e cols. (17) corroboram com a hipótese de que grandes centros urbanos passaram a experimentar a elevação dessa enfermidade mais precocemente do que outras capitais onde o processo de industrialização e urbanização ocorreu de forma mais tardia. Esses autores, estudando a evolução da mortalidade por diabetes melito no município de São Paulo, em uma série histórica de 79 anos (1900-1978), verificaram uma ascensão progressiva dos coeficientes até por volta de 1960 e posterior estabilização desses indicadores em valores próximos a 20 por 100 mil habitantes. Ao analisar a MP pela doença, verificaram que ela aumentou mais do que o coeficiente de mortalidade.

Laurenti e cols. (17) chamam a atenção, ainda, para o fato de que, no município de São Paulo, a mortalidade é mais elevada do que a observada em vários países, inclusive alguns desenvolvidos, onde, de maneira geral, as DCNT são mais frequentes como causa de morte.

Torna-se oportuno, também, destacar, em capitais como São Paulo, o efeito das correntes migratórias na composição etária da população, influenciando, dessa forma, o aumento da longevidade e, por conseguinte, o aumento de DCNT. Braga (18) refere que, durante a década de 1950, foram registradas as maiores taxas de migração interna da história do país, acompanhando os mesmos movimentos que se desenhavam nas décadas anteriores, quando as capitais Rio de Janeiro (RJ) e São Paulo figuravam como os dois maiores centros de atração dos migrantes originários principalmente dos Estados do Nordeste e Leste. Não menos importante é considerar o efeito da metropolização adiantada, tal como ocorre em São Paulo e outras capitais, como Rio de Janeiro, Belo Horizonte, Salvador e Recife.
Apesar da magnitude e da importância crescente do DMT2 nas capitais do país, esses dados ainda podem estar subestimados, uma vez que, de modo geral, os dados de mortalidade obtidos mediante atestados de óbito subestimam a importância do diabetes como causa associada e, frequentemente, essa enfermidade não aparece no atestado de óbito. Isso ocorre principalmente em relação aos idosos, nos quais estão presentes várias doenças crônicas, conforme referem Laurenti e cols. (17) e Franco (3).

Dessa forma, ao analisar a mortalidade segundo causas múltiplas, Laurenti e cols. (17) observaram que as causas básicas de morte mais frequentes entre os diabéticos são as doenças cardiovasculares. Entre elas, a mais importante é a doença isquêmica do coração (DIC), vindo seguida das doenças cerebrovasculares (DCbV), o que explica, de alguma forma, a importância dessas duas causas no padrão de morbimortalidade brasileiro. Por fim, referem que a análise das associações do diabetes melito com outras doenças, independentemente de ser causa básica ou não, revelou, novamente, que as doenças cardiovasculares são as que mais frequentemente se associam; neste caso, assumem papel de destaque as doenças hipertensivas, além das DIC, DCbV e outras doenças cardiovasculares; são igualmente relevantes as associações às doenças do aparelho respiratório, geniturinário, neoplasmas malignos e doenças do aparelho digestivo.

Os poucos e localizados estudos de tendência histórica de mortalidade por DCNT, com resgate de dados anteriores à série já disponibilizada pelo Ministério da Saúde, a partir 1980, justificam a realização de estudos panorâmicos nesse momento em que ainda são insuficientes àqueles que mostram o padrão de mortalidade por diabetes melito considerando o conjunto das capitais brasileiras. Nesse contexto, é necessário buscar possíveis particularidades na conformação e no desenvolvimento experimentado pelas capitais brasileiras, contemplando na análise a influência de fatores socioambientais existentes entre as macrorregiões brasileiras às quais pertencem.

O comportamento das RMP por diabetes melito no período considerado neste estudo sugere que essa enfermidade vem crescendo em importância epidemiológica, acompanhando o aumento da industrialização verificado a partir da década de 1930 e no pós-guerra no Brasil, a exemplo de São Paulo, que influenciou sobremaneira as condições e o estilo de vida da população (19). 
No que se refere ao enfrentamento desse contexto, torna-se indispensável considerar a heterogeneidade cultural, demográfica e socioeconômica, às quais estão submetidas as populações das regiões brasileiras. Também são diferentes nessas regiões a qualidade da assistência prestada, a capacidade diagnóstica e a qualidade das informações fornecidas.

Agradecimentos: ao Professor Ruy Laurenti, do Centro Colaborador da Organização Mundial da Saúde (OMS) para a Classificação de Doenças no Brasil, da Faculdade de Saúde Pública da Universidade de São Paulo, pelo apoio na compatibilização das nomenclaturas e códigos dos grupos, subgrupos e causas de óbitos por ano de revisão da CID no período de 1950 a 2000.

Declaração: os autores declaram não haver conflitos de interesse científico neste estudo.

\section{REFERÊNCIAS}

1. Santos M. A urbanização brasileira. 2 ed. São Paulo: Hucitec; 1994.

2. Lima-Costa MF, Veras R. Saúde pública e envelhecimento. Cad Saúde Pública. 2003;19(3):700-1.

3. Franco LJ. Epidemiologia do diabetes mellitus. In: Lessa I. O adulto brasileiro e as doenças da modernidade: epidemiologia das doenças crônicas não-transmissíveis. São Paulo/Rio de Janeiro: Hucitec/Abrasco; 1998. p. 123-37.

4. Fontbonne, A. A síndrome de resistência à insulina e complicações vasculares do diabetes mellitus não insulino-dependente. Diabetes \& Metab. 1997;1:11-9

5. Fontbonne A, Freese E. Epidemiologia do diabetes tipo 2 e da resistência à insulina. In: Freese $E$, org. Epidemiologia, políticas $e$ determinantes das doenças crônicas não transmissíveis no Brasil. Recife: Editora Universitária da UFPE; 2006. p. 157-75.

6. King H, Aubert RE, Herman WH. Global burden of diabetes, 19952025: prevalence, numeric estimates and projections. Diabetes Care. 1998;21(9):1414-31.

7. Expert Commitee on the Diagnosis and Classification of Diabetes Mellitus. Diabetes Care, Alexandria. 1997;20(7):1183-97.

8. Malerbi DA, Franco LJ. Multicenter study of the prevalence of diabetes mellitus and impaired glucose tolerance in the urban
Brazilian population aged 30-69 years. The Brazilian cooperative group on the study of diabetes prevalence. Diabetes Care, Alexandria. 1992;15:1509-16.

9. Brasil. Secretaria de Políticas Públicas. Plano de reorganização da atenção à hipertensão arterial e ao diabetes mellitus. Rev Saúde Pública. 2001;35(6):585-8.

10. IBGE. Estatísticas do século XX. Rio de Janeiro: Centro de Documentação e Disseminação de Informações, IBGE; 2003. 1 CDROM.

11. Coordenação Geral de Doenças e Agravos não Transmissíveis. Síntese da oficina de vigilância em doenças crônicas não transmissíveis. Ciências e Saúde Coletiva. 2004;9(4):957-62.

12. Laurenti R, Mello Jorge MHP, Gotlieb SLD. A confiabilidade dos dados de mortalidade e morbidade por doenças crônicas não transmissiveis. Ciência e Saúde Coletiva. 2004;9(4):900-20.

13. Zimmet P, Albert KG, Shaw J. Global and societal implications of the diabetes epidemic. Nature. 2001;414(6865):782-7.

14. Popkin BM, Gordon-Larsen P. The nutrition transition: Worldwide obesity dynamics and their determinants. Int J Obes Relat Metab Disord. 2004;28 Suppl 3:S2-9.

15. Brasil. Ministério da Saúde. Departamento de Informática do SUS. Sistema de informação de mortalidade. Disponível em: $<$ http://tabnet.datasus.gov.br/cgi/deftohtm.exe?sim/cnv/obtbr. def>. Acesso em: 28 ago. 2006.

16. Freese $E$, Fontbonne A. Transição epidemiológica comparada: modernidade, precariedade e vulnerabilidade. In: Freese E, org. Epidemiologia, políticas e determinantes das doenças crônicas não transmissíveis no Brasil. Recife: Editora Universitária da UFPE; 2006. p. 17-46.

17. Laurenti R, Fonseca LAM, Costa Jr ML. Mortalidade por diabetes mellitus no município de São Paulo (Brasil). Evolução em um período de 79 anos (1900-1978) e análise de alguns aspectos sobre associação de causas. Rev Saúde Pública. 1982;16:77-91.

18. Braga FG. Migração interna e urbanização no Brasil contemporâneo: um estudo da rede de localidades centrais do Brasil (1980/2000). In: Encontro Nacional de Estudos Populacionais, 15. 2006, Caxambu, MG. [Trabalhos apresentados...]. Belo Horizonte: ABEP, 2006. Disponível em: <http://www.abep.nepo.unicamp.br/ encontro 2006/docspdf/ABEP2006_573.pdf>. Acesso em: $15 \mathrm{dez}$. 2006.

19. Cesse EAP. Epidemiologia e determinantes sociais das doenças crônicas não transmissíveis no Brasil. 2007. Tese [Doutorado em Saúde Pública]. Recife: Centro de Pesquisas Aggeu Magalhães, Fundação Oswaldo Cruz; 2007. 